\title{
Evaluation of magnetic helicity density in the wave number domain using multi-point measurements in space
}

\author{
Y. Narita ${ }^{1}$, G. Kleindienst ${ }^{1}$, and K.-H. Glassmeier ${ }^{1,2}$ \\ ${ }^{1}$ Institut für Geophysik und extraterrestrische Physik, Technische Universität Braunschweig, Mendelssohnstr. 3, \\ 38106 Braunschweig, Germany \\ ${ }^{2}$ Max-Planck-Institut für Sonnensystemforschung, Max-Planck-Straße 2, 37191 Katlenburg-Lindau, Germany
}

Received: 13 July 2009 - Revised: 21 September 2009 - Accepted: 14 October 2009 - Published: 21 October 2009

\begin{abstract}
We develop an estimator for the magnetic helicity density, a measure of the spiral geometry of magnetic field lines, in the wave number domain as a wave diagnostic tool based on multi-point measurements in space. The estimator is numerically tested with a synthetic data set and then applied to an observation of magnetic field fluctuations in the Earth foreshock region provided by the four-point measurements of the Cluster spacecraft. The energy and the magnetic helicity density are determined in the frequency and the wave number domain, which allows us to identify the wave properties in the plasma rest frame correcting for the Doppler shift. In the analyzed time interval, dominant wave components have parallel propagation to the mean magnetic field, away from the shock at about Alfvén speed and a left-hand spatial rotation sense of helicity with respect to the propagation direction, which means a right-hand temporal rotation sense of polarization. These wave properties are well explained by the right-hand resonant beam instability as the driving mechanism in the foreshock. Cluster observations allow therefore detailed comparisons with various theories of waves and instabilities.
\end{abstract}

Keywords. Interplanetary physics (Interplanetary magnetic fields) - Space plasma physics (Experimental and mathematical techniques; Waves and instabilities)

\section{Introduction}

Magnetic helicity is one of the invariants (time- and gaugeindependent) in ideal magnetohydrodynamics and it is given as an integral of the product of the vector potential $\boldsymbol{A}$ and the magnetic field $\boldsymbol{B}$ over a volume $V$,

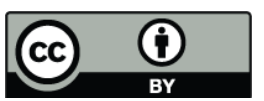

Correspondence to: Y. Narita (y.narita@tu-bs.de)
$H^{M}=\int \boldsymbol{A} \cdot \boldsymbol{B} d V$

It is a measure of the topological property of magnetic field lines (Berger and Field, 1984). In a plasma the magnetic field lines often take a spiral form as is often seen in coronal mass ejections and interplanetary magnetic clouds. Some of the electromagnetic waves also exhibit helical field lines about its propagation direction. Magnetic helicity also plays a key role in theoretical treatments of plasma dynamics. For example, the force-free magnetic field geometry is one of the minimum energy state under the conserved magnetic helicity (Woltjer, 1958); the treatment of absolute equilibrium state suggests a possibility of inverse cascade process of the magnetic helicity in turbulence (Frisch et al., 1975; Biskamp, 2003). The magnetic helicity can also be used as a diagnostic tool in the study of magnetic reconnection (Wiegelmann and Büchner, 2001, 2002).

In contrast to its importance in plasma dynamics, a proper determination of the magnetic helicity using spacecraft observations is still a challenge for several reasons. One is that the vector potential must be determined from the measurement, which requires the knowledge of the electric current distribution in space. Another difficulty is that the integral should be performed over a volume bounded by the magnetically closed surface on which the magnetic field is perpendicular to the surface normal direction, otherwise the magnetic helicity is not any more time-independent nor gaugeindependent in the system. However, it is presented in this paper that the density of the magnetic helicity $h=\boldsymbol{A} \cdot \boldsymbol{B}$ can be still experimentally determined under certain conditions and furthermore it is demonstrated that the helicity density serves as a useful wave diagnostic tool in spacecraft observations. It is worthwhile to note that the determination of the helicity density can be performed without using gauge-invariant vector potential if we assume that the magnetic field consists of

Published by Copernicus Publications on behalf of the European Geosciences Union. 
a weakly inhomogeneous background and a random smallscale magnetic field (Subramanian and Brandenburg, 2006), while in our method the helicity density is determined for magnetic field fluctuations using the vector potential.

It is known that electromagnetic waves in general have two degrees of freedom in polarization perpendicular to its propagation direction. Waves can be linearly, elliptically or circularly polarized. The term "polarization" for waves in plasma is defined such that right- or left-handed polarization has the sense of rotation of a fluctuating field vector in time at a fixed point in space, when viewed in the direction parallel to the magnetic field at positive frequency (Stix, 1962). Under this definition, a right-hand polarized wave propagating either parallel or anti-parallel to the mean magnetic field $\boldsymbol{B}_{0}$ possesses fluctuating field vectors that rotate in the same sense as the gyro-motion of an electron. Similarly, a lefthand polarized wave rotates in the same sense as a gyrating ion.

Helicity, in contrast to the concept of polarization, is a measure of the sense of rotation in the spatial domain at a fixed time point. The positive helicity is defined to be a lefthand sense of rotation with respect to the wave vector (or the propagation direction), while the negative helicity implies a right-handed field structure, see for example, Smith et al. (1983); Glassmeier et al. (1989); Gary (1993).

Polarization and helicity are different representations of wave structure and closely related to each other. For example, a wave propagating parallel to the mean magnetic field with a positive helicity is represented as a right-hand polarized wave in the time domain (Fig. 1 top panels). It is worthwhile to note that there is an ambiguity when relating polarization with helicity, that is a wave propagating anti-parallel to the mean magnetic field with a negative helicity also exhibits right-hand polarization in the time domain (Fig. 1 bottom panels). It is possible to determine the polarization property using a single point measurement in space, but it is not possible to determine the wave helicity uniquely because the single point measurement does not provide the propagation direction of waves in general.

Here we point out that the multi-point measurements of the magnetic fields in space such as the Cluster mission (Balogh et al., 2001; Escoubet et al., 2001) or the Themis mission (Angelopoulos, 2008; Auster et al., 2008) have the potential to determine the magnetic helicity density. We develop an estimator of the magnetic helicity density in the wave number domain and present a numerical test as well as an application to wave analyses in the Earth foreshock region ahead of the bow shock using Cluster data.

\section{Estimator of magnetic helicity density}

There are two tasks in order to construct an estimator for the magnetic helicity density. The first is to estimate the vector potential $\boldsymbol{A}$. This can be done by uncurling the definition of the vector potential, which allows us to express the vector potential explicitly as a function of the wave vector and the amplitude of magnetic field fluctuation. The second task is to estimate the amplitude as a function of the wave vector. This can be accomplished by the use of multi-point measurements.

\subsection{Estimating vector potential}

The vector potential for a fluctuating magnetic field can be obtained by uncurling the definition of the vector potential

$\boldsymbol{B}=\nabla \times \boldsymbol{A}$.

We multiply Eq. (2) by the curl operator,

$\nabla \times(\nabla \times \boldsymbol{A})=\nabla(\nabla \cdot \boldsymbol{A})-\nabla^{2} \boldsymbol{A}$.

Here the first term on the right hand side vanishes under the Coulomb gauge

$\nabla \cdot \boldsymbol{A}=0$,

in which case we obtain

$\nabla \times \boldsymbol{B}=-\nabla^{2} \boldsymbol{A}$.

For a plane wave geometry $\boldsymbol{B}=\boldsymbol{b} \exp [i \boldsymbol{k} \cdot \boldsymbol{r}]$ and

$\boldsymbol{A}=\boldsymbol{a} \exp [i \boldsymbol{k} \cdot \boldsymbol{r}]$ Eq. (5) becomes

$i \boldsymbol{k} \times \boldsymbol{b}=-k^{2} \boldsymbol{a}$,

where $k=|\boldsymbol{k}|$. The vector potential amplitude $\boldsymbol{a}$ is therefore given as

$\boldsymbol{a}=-\frac{i}{k^{2}} \boldsymbol{k} \times \boldsymbol{b}$.

The magnetic helicity density can be determined by building a product between $\boldsymbol{a}$ and $\boldsymbol{b}$,

$h=\left\langle\boldsymbol{a}^{\dagger} \cdot \boldsymbol{b}\right\rangle$,

where the angular bracket denotes the operation of averaging either in the time domain or in the frequency domain. Using the expression of the vector potential $\boldsymbol{a}$ (Eq. 7), the estimator for the helicity density is given as a combination of cross correlation of magnetic field fluctuations between different components:

$$
\begin{aligned}
\left\langle\boldsymbol{a}^{\dagger} \cdot \boldsymbol{b}\right\rangle= & -\frac{i}{k^{2}}\left[k_{x}\left(\left\langle b_{y}^{*} b_{z}\right\rangle-\left\langle b_{z}^{*} b_{y}\right\rangle\right)+k_{y}\left(\left\langle b_{z}^{*} b_{x}\right\rangle-\left\langle b_{x}^{*} b_{z}\right\rangle\right)\right. \\
& \left.+k_{z}\left(\left\langle b_{x}^{*} b_{y}\right\rangle-\left\langle b_{y}^{*} b_{x}\right\rangle\right)\right] .
\end{aligned}
$$

Here we define the helicity density such that the positive helicity $\left\langle\boldsymbol{a}^{\dagger} \cdot \boldsymbol{b}\right\rangle>0$ has a left-hand sense of rotation with respect to the wave vector direction. When viewed at a fixed point in space as time varies, it has the right-handed sense of polarization about the propagation direction. The dagger $\dagger$ and 
(a) Forward propagation, positive helicity
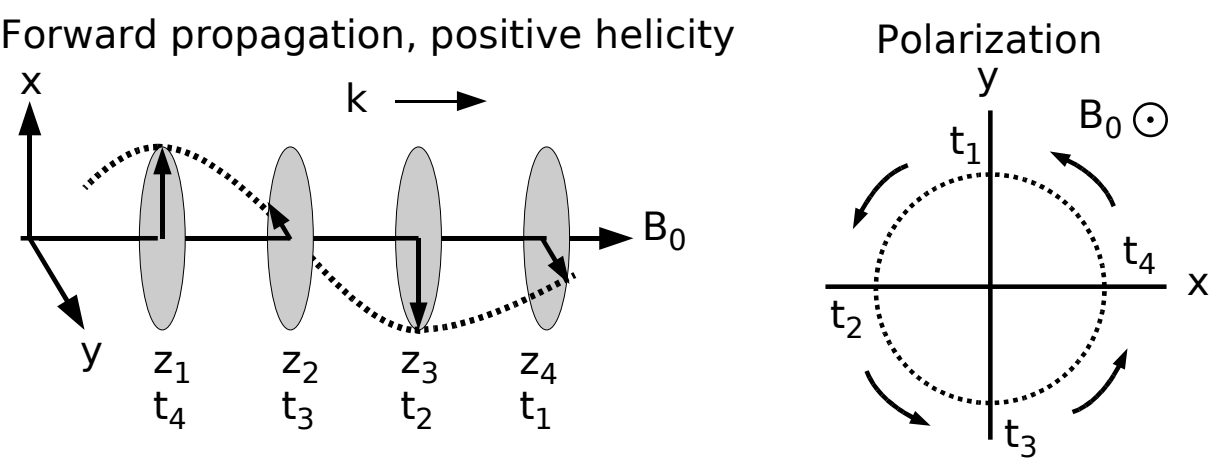

(b) Backward propagation, negative helicity

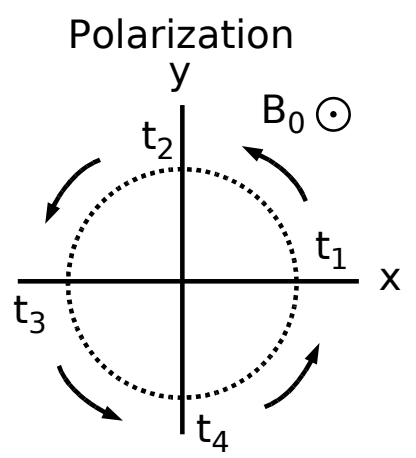

Fig. 1. (a) Wave propagating forward to the mean magnetic field $\left(\boldsymbol{B}_{0}\right)$ with a positive helicity, left-handed field rotation with respect to the propagation direction, corresponding to the field rotation with the order from $z_{1}$ to $z_{4}$ (top left panel), appears as right-hand polarization about the mean field in the time domain (corresponding to the order from $t_{1}$ to $t_{4}$ ). (b) Wave propagating backward to the mean field with a negative helicity also appears as right-hand polarization in the temporal domain.

the asterisk $*$ denote the Hermitian and the complex conjugate operation, respectively. Equation (9) implies also that the magnetic helicity density can be interpreted as a measure of asymmetry in cross correlation between $i$-th and $j$-th component of the magnetic field,

$$
P_{i j}(\boldsymbol{k})-P_{j i}(\boldsymbol{k})=\left\langle b_{i}^{*}(\boldsymbol{k}) b_{j}(\boldsymbol{k})\right\rangle-\left\langle b_{j}^{*}(\boldsymbol{k}) b_{i}(\boldsymbol{k})\right\rangle .
$$

The task to obtain the helicity density from the measurements now reduces to evaluate the amplitude or the correlation matrix in the wave vector domain.

\subsection{Estimating fluctuation amplitude}

The cross correlation can be determined by the four-point measurements of Cluster using the wave telescope technique (also referred to as the $k$-filtering), developed by Pinçon and Lefeuvre (1991); Motschmann et al. (1996); Glassmeier et al. (2001). In this method we construct a generalized cross spectral density (CSD) matrix in the frequency domain and project it into the wave vector domain using a suitable weight. It is also presented that this technique allows to determine various kinds of cross correlation, for example a bispectrum (a triple correlation) using the magnetic field and the electron density data serves as a diagnostic tool of wavewave interactions (Narita et al., 2008).

The CSD matrix is constructed from the measurements as

$\mathbf{M}(\omega)=\frac{1}{T}\left\langle\boldsymbol{S}(\omega) \boldsymbol{S}^{\dagger}(\omega)\right\rangle$

where $\omega$ denotes the angular frequency, $T$ the length of the measurement time. $\boldsymbol{S}(\omega)$ is the state vector of the measured magnetic field fluctuations that are Fourier transformed from the time domain to the frequency domain. Here the background field is assumed to be constant. In the case of the Cluster magnetic field measurement, the state vector consists of 12 elements ( 3 magnetic field components times 4 measurement points):

$\boldsymbol{S}(\omega)=\left(\begin{array}{l}\boldsymbol{B}_{1}(\omega) \\ \boldsymbol{B}_{2}(\omega) \\ \boldsymbol{B}_{3}(\omega) \\ \boldsymbol{B}_{4}(\omega)\end{array}\right)$,

where the subscript refers to the measurement point (i.e., spacecraft). The CSD matrix (Eq. 11) is projected into a $3 \times 3$ matrix using a dimensionless weight matrix $\mathbf{W}(\omega, \boldsymbol{k})$ :

$\mathbf{P}(\omega, \boldsymbol{k})=\mathbf{W}^{\dagger}(\omega, \boldsymbol{k}) \mathbf{M}(\omega) \mathbf{W}(\omega, \boldsymbol{k})$. 
Here we choose the minimum variance weight, which has the form

$\mathbf{W}(\omega, \boldsymbol{k})=\mathbf{M}^{-1}(\omega) \mathbf{H}(\boldsymbol{k}) \mathbf{V}(\boldsymbol{k})\left[\mathbf{V}^{\dagger}(\boldsymbol{k}) \mathbf{H}^{\dagger}(\boldsymbol{k}) \mathbf{M}^{-1}(\omega) \mathbf{H}(\boldsymbol{k}) \mathbf{V}(\boldsymbol{k})\right]^{-1}$,

where $\mathbf{H}(\boldsymbol{k})$ is a $12 \times 3$ matrix and called the steering matrix:

$\mathbf{H}(\boldsymbol{k})=\left(\begin{array}{l}\mathbf{I} \exp \left(i \boldsymbol{k} \cdot \boldsymbol{r}_{1}\right) \\ \mathbf{I} \exp \left(i \boldsymbol{k} \cdot \boldsymbol{r}_{2}\right) \\ \mathbf{I} \exp \left(i \boldsymbol{k} \cdot \boldsymbol{r}_{3}\right) \\ \mathbf{I} \exp \left(i \boldsymbol{k} \cdot \boldsymbol{r}_{4}\right)\end{array}\right)$

with $\mathbf{I}$ the $3 \times 3$ unit matrix. The steering matrix reflects the periodic spatial pattern characterized by the wave vector $\boldsymbol{k}$ with amplitude unity. The matrix $\mathbf{V}(\boldsymbol{k})$ in Eq. (14) is given as

$\mathbf{V}(k)=\mathbf{I}+\frac{k \boldsymbol{k}}{k^{2}}$,

where $k=|\boldsymbol{k}|$. The weight matrix (Eq. 14) is optimized to minimize the trace of the projected matrix (Eq. 13) under two constraints. The first is that the weight matrix satisfies the unit gain condition:

$\mathbf{W}^{\dagger}(\omega, \boldsymbol{k}) \mathbf{H}(\boldsymbol{k})=\mathbf{I}$.

The second is the divergence-free nature of the magnetic field, $\nabla \cdot \boldsymbol{B}=0$, which results in the matrix $\mathbf{V}(\boldsymbol{k})$. It is worthwhile to note that the weight matrix is determined by the measurement itself (the CSD matrix in Eq. 11). The matrix projected to the wave vector domain may be given analytically from Eq. (13) and (14) as

$\mathbf{P}(\omega, \boldsymbol{k})=\left[\mathbf{V}^{\dagger}(\boldsymbol{k}) \mathbf{H}^{\dagger}(\boldsymbol{k}) \mathbf{M}^{-1}(\omega) \mathbf{H}(\boldsymbol{k}) \mathbf{V}(\boldsymbol{k})\right]^{-1}$.

This estimator of $\mathbf{P}(\omega, \boldsymbol{k})$ is the essence of the wave telescope or $k$-filtering technique (Pinçon and Lefeuvre, 1991; Motschmann et al., 1996; Glassmeier et al., 2001), which was developed particularly for the multi-point measurements of the Cluster mission. Note that the projection in Eq. (13) does not change the units of the matrix elements, and therefore the projected matrix $\mathbf{P}(\omega, \boldsymbol{k})$ has the same unit as that of the CSD matrix (Eq. 11), for example $\mathrm{nT}^{2} / \mathrm{Hz}$ in the case of the magnetic field (the same dimension as the spectral density in the frequency domain). The trace of the projected matrix gives an estimate of the fluctuation energy at a given set of frequency and wave vector. The cross correlation matrix in the wave vector domain is obtained by integrating the matrix $\mathbf{P}(\omega, \boldsymbol{k})$ over the frequency,

$P_{i j}(\boldsymbol{k})=\int P_{i j}(\omega, \boldsymbol{k}) d \omega$,

which is given in units of squared amplitude $\left(\mathrm{nT}^{2}\right)$.

\section{Applications}

\subsection{Analytical model}

For a spiral magnetic field geometry it is possible to express the magnetic helicity density analytically. The spiral magnetic field can be expressed as

$\boldsymbol{B}=\left(\begin{array}{c}\delta B_{x} e^{i k_{z} z} \\ \delta B_{y} e^{i\left(k_{z} z \pm \pi / 2\right)} \\ B_{0}\end{array}\right)$,

where $\delta B_{x}$ and $\delta B_{y}$ denote the fluctuation amplitudes and they are positive finite. The sign in front of the phase $\pi / 2$ in the $B_{y}$ component denotes the sense of rotation such that the minus and the plus sign give the left-hand and the right-hand spatial rotation about the $\mathrm{z}$-axis, respectively. The wave vector has only the z-component parallel to the mean magnetic field direction,

$\boldsymbol{k}=\left(\begin{array}{c}0 \\ 0 \\ k_{z}\end{array}\right)$.

It is straightforward to calculate the magnetic helicity density using Eq. (9),

$h= \pm \frac{2}{k_{z}} \delta B_{x} \delta B_{y}$.

This estimator can be used to evaluate the magnetic helicity density for single spacecraft measurements (Matthaeus and Goldstein, 1982; Matthaeus et al., 1982; Glassmeier et al., 1989). In a supersonic or super-Alfvénic flow such as the solar wind the temporal variation in the measurement reasonably reflects the spatial variation along the flow velocity and Taylor's hypothesis can be used, that is relabeling the frequency as the wave number,

$\omega_{s c}=k V_{f}$

where $V_{f}$ denotes the flow speed. However, it should be noted that one cannot distinguish between two propagation directions in the single point measurements, i.e., if the wave number is positive or negative and therefore the estimate of the helicity density reflects essentially polarization and the ambiguity remains about the sign of the wave helicity as shown in Fig. 1.

\subsection{Numerical test}

The estimator Eq. (9) is numerically tested using an artificially generated data set. We put two wave components on a mean magnetic field $B_{0}=10.0 \mathrm{nT}$ in the z-direction. The first wave component has the following properties: forward propagation to the mean field, wavelength $604 \mathrm{~km}$, period $30 \mathrm{~s}$, positive helicity with amplitudes $\delta B_{x}=2.5 \mathrm{nT}$ 

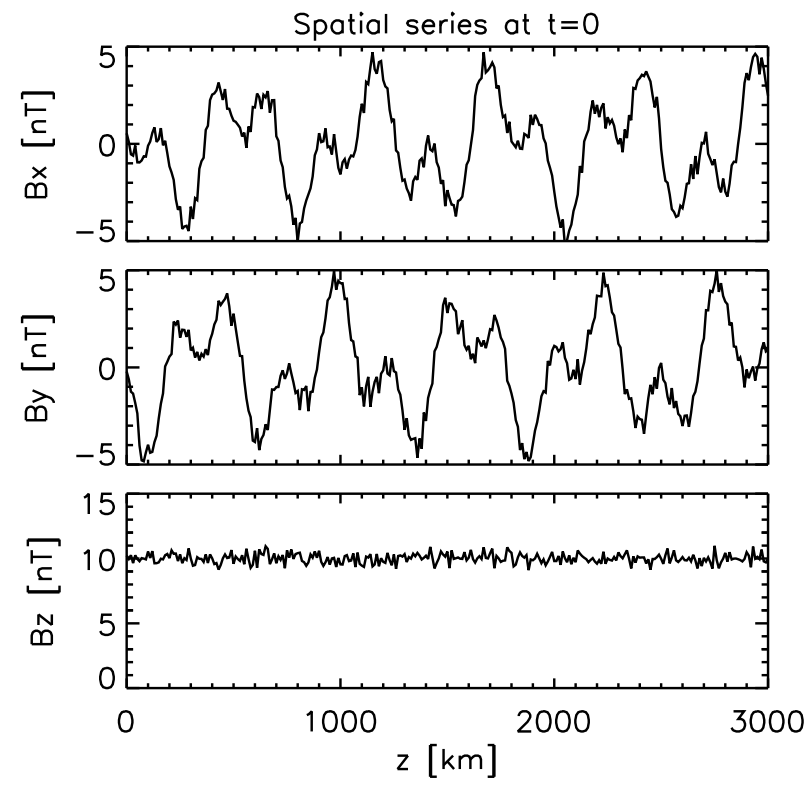

Fig. 2. Magnetic field data that are numerically generated and used for the test of the estimator of the magnetic helicity density. The plot displays spatial variation of the magnetic field at the initial time $t=0$.

and $\delta B_{y}=2.5 \mathrm{nT}$. The second component has the following properties: backward propagation, wavelength $253 \mathrm{~km}$, period $8 \mathrm{~s}$, negative helicity with amplitude $\delta B_{x}=2.0 \mathrm{nT}$ and $\delta B_{y}=2.0 \mathrm{nT}$. In addition to the two wave components we put a random fluctuation field as noise in the observation when generating the time series data of the wave form. The spatial variation of the generated magnetic field is displayed in Fig. 2 at the initial time $t=0 \mathrm{~s}$. The $\delta B_{x}$ and $\delta B_{y}$ components show the superposed wave field in the range from $-5 \mathrm{nT}$ to $5 \mathrm{nT}$, whereas the $B_{z}$ component exhibits the mean magnetic field with noise. The magnetic field fluctuation is then sampled at four different points (virtual sensors) on the z-axis: $z_{1}=100 \mathrm{~km}, z_{2}=180 \mathrm{~km}, z_{3}=290 \mathrm{~km}, z_{4}=375 \mathrm{~km}$. The distance between the sensors is chosen not to be exactly regular and small enough to resolve the wavelengths of the generated waves.

Figure 3 displays the magnetic field measured at the position of sensor $1\left(z_{1}=100 \mathrm{~km}\right)$. The two waves can be identified as different periods in the $B_{x}$ and $B_{y}$ components, while the $B_{z}$ component exhibits merely the mean magnetic field. The magnetic field measured at the other sensor positions $\left(z_{2}\right.$ to $z_{4}$ ) also exhibit the same characteristics (fluctuations in the $\mathrm{x}$ - and $\mathrm{y}$-component with two different periods) but the wave phases are different at the four sensor positions.

In the analysis the wave telescope technique is extensively used. The fluctuation energy is investigated by determining the trace of the projected CSD matrix $\mathbf{P}(\omega, \boldsymbol{k})$ at various frequencies and wave numbers in the mean field direction. The energy distribution in the frequency and the wave

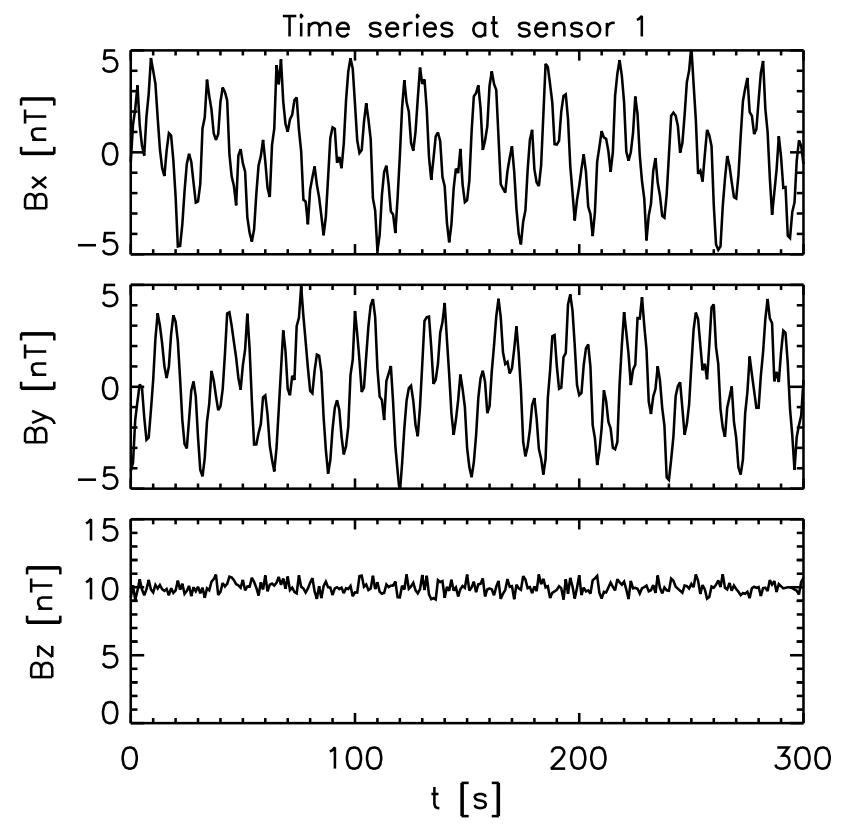

Fig. 3. Generated magnetic field data used for the test of the helicity estimator. The plot displays temporal variation of the magnetic field at sensor 1 .

number domain (hereafter, the $\omega-k$ domain) is displayed in Fig. 4 top panel. Two peaks can be identified in the energy distribution, one around the frequency $f=0.033 \mathrm{~Hz}$ (period $30 \mathrm{~s}$ ) and the wave number $k=0.010 \mathrm{rad} / \mathrm{km}$ (wavelength $604 \mathrm{~km}$ in the positive z-direction), and another peak around the frequency $f=0.125 \mathrm{~Hz}$ (period $8 \mathrm{~s}$ ) and the wave number $k=-0.025 \mathrm{rad} / \mathrm{km}$ (wavelength $253 \mathrm{~km}$ in the negative $\mathrm{z}-$ direction). The two peaks represent properly the frequencies and the wave numbers of the generated waves.

The estimator of the magnetic helicity density is then applied to the test data set, which also makes use of the wave telescope technique. Figure 4 middle panel displays the magnitude of the helicity density in the $\omega-k$ domain. Again, two peaks can be identified in the helicity density at the frequencies and the wave numbers used for the synthetic data set. The helicity density is then summed over the frequencies. The two wave components can again be identified in the one-dimensional helicity density distribution (Fig. 4 bottom) at the wave numbers $k=0.010 \mathrm{rad} / \mathrm{km}$ and $k=-0.025 \mathrm{rad} / \mathrm{km}$, respectively. The determined helicity densities are $h=378.9 \mathrm{nT}^{2} \mathrm{~km}$ and $h=-47.8 \mathrm{nT}^{2} \mathrm{~km}$ at the former and the latter wave numbers. For comparison, the analytical model in the previous subsection yields the helicity density $h=1201.6 \mathrm{nT}^{2} \mathrm{~km}$ and $h=-322.2 \mathrm{nT}^{2} \mathrm{~km}$ for the former and the latter wave components, respectively. Therefore the helicity densities estimated using the wave telescope technique are somewhat lower than that of the analytical model. 

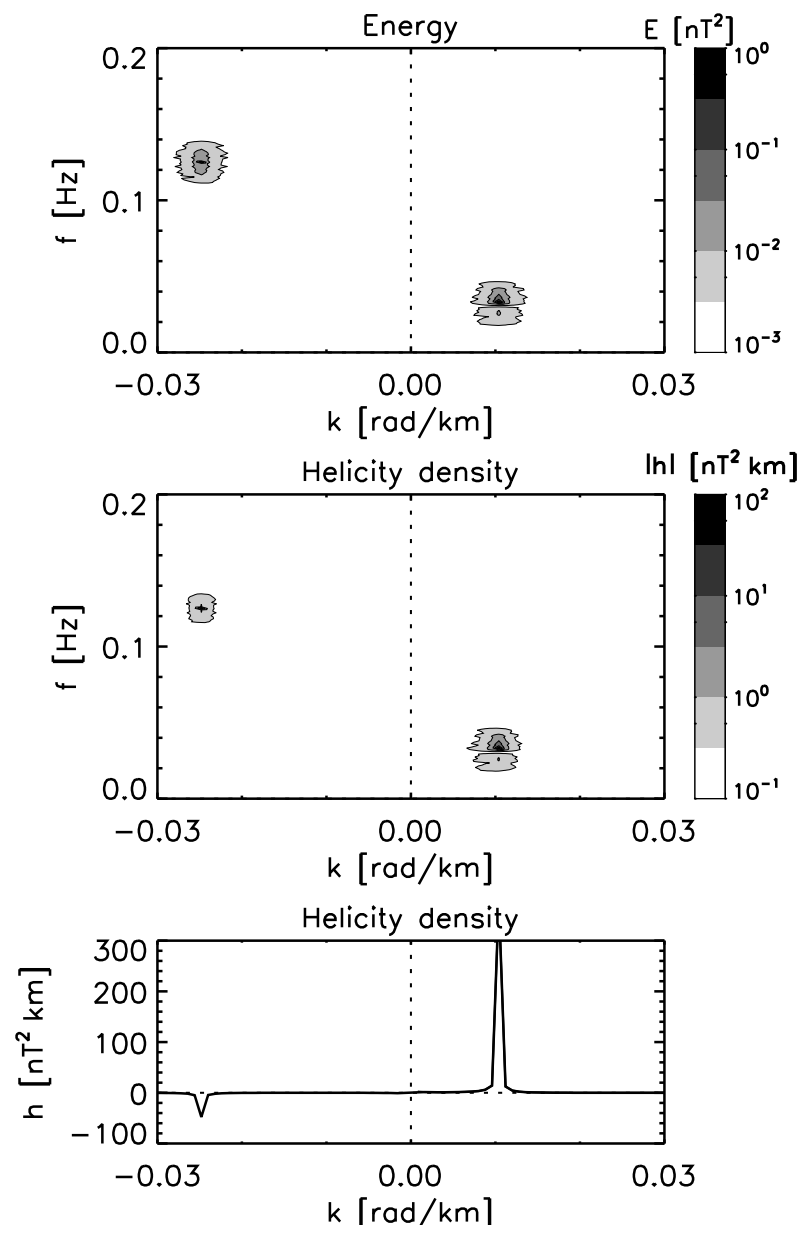

The background level is much lower and the peak is clearer for the data set with $10 \%$ noise level. Table 1. summarizes the energy, the helicity density, and their respective ratios to the background levels at the wave number of the first wave component, $0.010 \mathrm{rad} / \mathrm{km}$. The background levels are measured at the wave number $0.004 \mathrm{rad} / \mathrm{km}$. We find the following features in the results.

(1) The peak can be identified even under $200 \%$ noise level in the both energy and helicity density. In our tests the peak can be identified up to the noise amplitude 5 times as large as the signal amplitude (500\% noise amplitude). In other

Fig. 4. Energy distribution (top) and magnetic helicity density distribution (middle) in the frequency and wave number domain derived from the test data set. The bottom panel displays the helicity averaged over the frequencies. The wave number is parallel to the mean magnetic field.

\section{Noise and sampling effects}

The numerical test is then performed under different conditions to see (1) effects of noise in the data set and (2) effects of time series sampling. For the noise effects we generate synthetic data with the noise amplitude from $0 \%$ to $200 \%$ to that of the first wave component, $2.5 \mathrm{nT}$. The data are sampled with the rate $1 \mathrm{~s}$ at four sensor positions and the energy and the helicity density are estimated from the four time series data sets. Figure 5 displays two examples of the determined energy and helicity density in the wave number range between $0.000 \mathrm{rad} / \mathrm{km}$ and $0.020 \mathrm{rad} / \mathrm{km}$ for the noise level $10 \%$ (noise amplitude $0.25 \mathrm{nT}$, solid lines) and 100\% (amplitude $2.5 \mathrm{nT}$, dotted lines) in the top and bottom panels, respectively. Both the energy and the helicity density curves show a peak at the right wave number $(0.010 \mathrm{rad} / \mathrm{km})$, but the background levels are different between the two data sets. words, our method can detect a helical wave even if the signal amplitude is only $20 \%$ of that of noise. Of course, the background level increases for larger noise amplitudes in the spectra. (2) If the noise is not given in the data set, the estimator gives a smaller value of the energy and the helicity density. This effect stems from the inversion of the CSD matrix in Eq. (18), that is the matrix cannot be inverted for a data set with a pure plane wave because at least one eigenvalue in the matrix is very small in such a data set and the inversed matrix diverges (Vogt et al., 2008). (3) Estimate of the helicity density is more stable than that of the energy against noise. While the energy exhibits a monotonous increase for larger noise levels, the helicity density is almost constant above the noise level $10 \%$. Also, the ratio to the background level is larger in the helicity density, which means a clearer peak in the spectrum.

For the investigation of the sampling effects we perform the same numerical test for different sampling rates $\Delta t=0.5 \mathrm{~s}, 1 \mathrm{~s}, 2 \mathrm{~s}$ and with the fixed time length $T=1024 \mathrm{~s}$ (Table 2). We find that the estimated energy and helicity density become diminished for higer sampling rates and that the ratio to the background level becomes larger, i.e. the peak is clearer. The reason for larger values of the energy and 
Table 1. Estimated energy and its ratio to the background level, and helicity density and its ratio to the background under various conditions of noise in the synthetic data sets.

\begin{tabular}{rrrrr}
\hline Noise $(\%)$ & $E\left(\mathrm{nT}^{2}\right)$ & $E / E_{0}$ & $h\left(\mathrm{nT}^{2} \mathrm{~km}\right)$ & $h / h_{0}$ \\
\hline 0 & 0.076 & $1.94 \times 10^{5}$ & 7.32 & $3.60 \times 10^{5}$ \\
1 & 1.24 & $2.28 \times 10^{4}$ & 119.4 & $6.98 \times 10^{4}$ \\
10 & 3.79 & $7.56 \times 10^{3}$ & 363.6 & $3.84 \times 10^{3}$ \\
50 & 4.01 & 33.4 & 379.1 & 234.4 \\
100 & 4.40 & 9.14 & 379.3 & 72.6 \\
200 & 5.74 & 3.02 & 378.2 & 24.8 \\
\hline
\end{tabular}

Table 2. Energy and helicity density analysis with different sampling rates.

\begin{tabular}{ccc}
\hline$\Delta t(\mathrm{~s})$ & $E\left(\mathrm{nT}^{2}\right)$ & $h\left(\mathrm{nT}^{2} \mathrm{~km}\right)$ \\
\hline 2 & 6.70 & 629.1 \\
1 & 4.08 & 381.1 \\
0.5 & 3.71 & 351.1 \\
\hline
\end{tabular}

the helicity for lower sampling rates is that their spectra are broader around the peak when the analysis is performed with a smaller number of data points. The wave telescope estimator is based on a minimization procedure of the power and the minimization works better with a larger number of data points. The energy distribution near the peak (particularly in the frequency domain) gives a larger value of the energy. Figure 6 top panel compares the helicity density in the two cases, $\Delta t=0.5 \mathrm{~s}$ and $2 \mathrm{~s}$. The peak is clearer with a moderate decrease for a higher sampling rate. We also perform the numerical test for different time length $(T=512 \mathrm{~s}, 1024 \mathrm{~s}$, and $2048 \mathrm{~s}$ ) with the fixed sampling rate $\Delta t=1 \mathrm{~s}$. The results are very similar to the investigation of the different sampling rates, that is the peak becomes clearer for a longer time interval in the analysis, while the peak value itself becomes moderately diminished (Table 3 and Fig. 6 bottom panel).

To summarize the analysis with the synthetic data, (1) the energy and the helicity density can be determined in the frequency and wave number domain using four point measurements; (2) the analysis method provides reasonable results even under a high noise level; (3) but estimated energy and helicity density are moderately dependent on how the statistical analysis is done (total time length $T$ and sampling rate $\Delta t)$

\subsection{Cluster spacecraft in the foreshock region}

The analysis method is then applied to the real spacecraft observation. We use the four-point magnetic field measurements of the Cluster spacecraft where the spacecraft encountered the Earth foreshock region (upstream of the Earth bow shock) from 08:10 to 08:30 UT on 18 February 2002, and de-

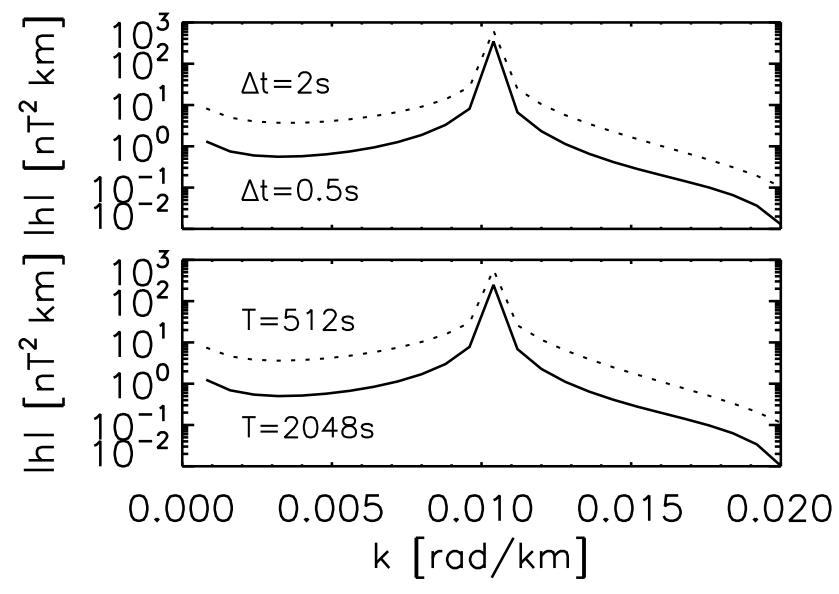

Fig. 6. Sampling effect analysis.

Table 3. Energy and helicity density analysis with different sampling time lengths.

\begin{tabular}{ccc}
\hline$T(\mathrm{~s})$ & $E\left(\mathrm{nT}^{2}\right)$ & $h\left(\mathrm{nT}^{2} \mathrm{~km}\right)$ \\
\hline 512 & 6.18 & 578.6 \\
1024 & 4.08 & 381.1 \\
2048 & 2.67 & 251.4 \\
\hline
\end{tabular}

tected large amplitude waves in the magnetic field. Figure 7 displays the time series of magnetic field data provided by Cluster-1 spacecraft in this time interval. The coordinate system in the plot is the mean-field aligned coordinate, in which the $\mathrm{z}$-axis is parallel to the mean magnetic field direction and the $x z$-plane is spanned by the mean magnetic field and the plasma bulk flow direction. The bulk flow is provided by the ion measurements on board Cluster (Rème et al., 2001). Cluster forms an almost regular tetrahedron with the interspacecraft distance about $100 \mathrm{~km}$ in this time interval.

The foreshock region is of great interest for studying waves in space plasma. The collisionless nature of the Earth bow shock serves as a scatterer and reflector of the incoming solar wind particles (ions and electrons) as one of the shock dissipation mechanisms. The reflected ions become a backstreaming beam along the magnetic field against the solar wind flow and form an unstable two-beam distribution in the velocity domain together with the incoming solar wind flow. This beam component provides free energy for driving an instability and excites waves in front of the shock.

The investigation of the energy and the helicity density with the Cluster data is performed in the plasma rest frame of reference. This is a co-moving frame with the mean plasma flow. Transformation from the spacecraft to the plasma rest frame is made by the Doppler shift equation,

$\omega_{r e}=\omega_{s c}-\boldsymbol{k} \cdot \boldsymbol{V}_{f}$. 

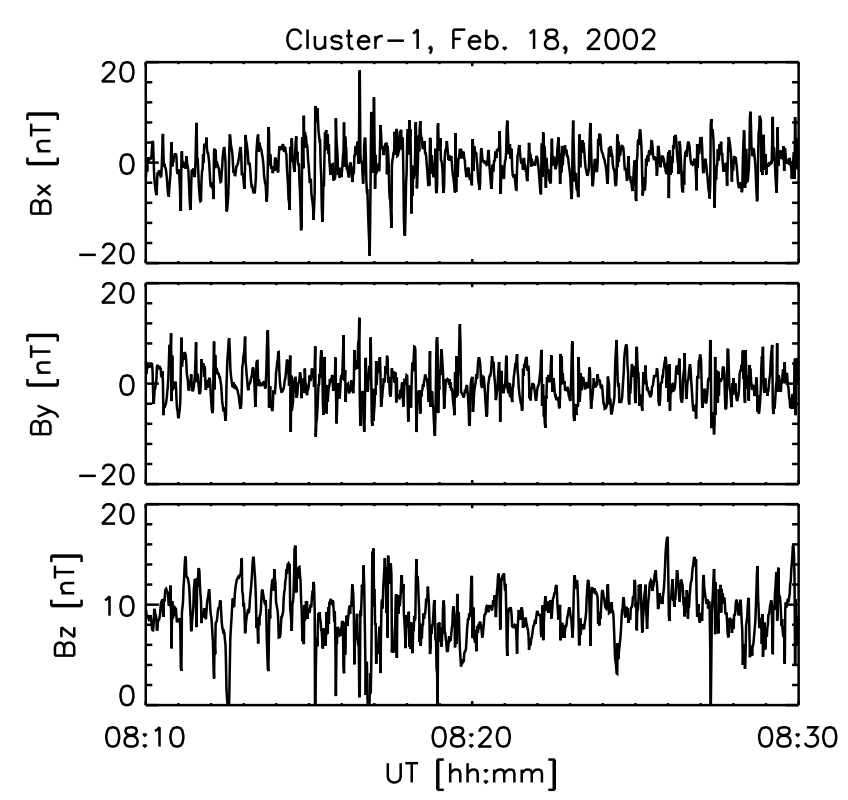

Fig. 7. Time series of magnetic field data observed by Cluster-1 in the Earth foreshock region. The data are presented in the mean field aligned coordinate system. The z-axis is aligned with the mean field direction and the xz-plane is spanned by the plasma flow direction and the mean magnetic field direction.

Here $\omega_{r e}$ and $\omega_{s c}$ denote the angular frequency in the plasma rest frame and the spacecraft frame, respectively. The mean flow velocity $\boldsymbol{V}_{f}$ is determined by the measurement of the bulk velocity using the electrostatic particle analyzer on board Cluster (CIS-HIA, Rème et al., 2001). We restrict our analysis in the positive frequency regime such that the direction of the wave vector agrees with the wave propagation direction, and therefore the signs of the frequency and the wave vector are reversed when the rest-frame frequency becomes negative after the Doppler shift correction. The sign reversal does not alter the wave propagation speed and direction (the phase speed vector is the same before and after the sign reversal).

Figure 8 top panel displays the energy distribution in the $\omega-k$ domain in the plasma rest frame. The wave number is aligned with the mean magnetic field direction in this analysis and the mean magnetic field is established from the time average (constant field) without detrending. We note that detrending of the data may reduce low-wave-number power in an ad hoc fashion as discussed by Matthaeus and Goldstein (1982).

The mean field has mostly the sunward component, (8.1 nT sunward, $-3.8 \mathrm{nT}$ dawn-to-dusk, $2.1 \mathrm{nT}$ ecliptic north component) and earlier Cluster data analyses using the wave telescope technique identified that the waves propagate almost parallel to the mean magnetic field in the presented interval (Narita et al., 2004; Narita and Glass-
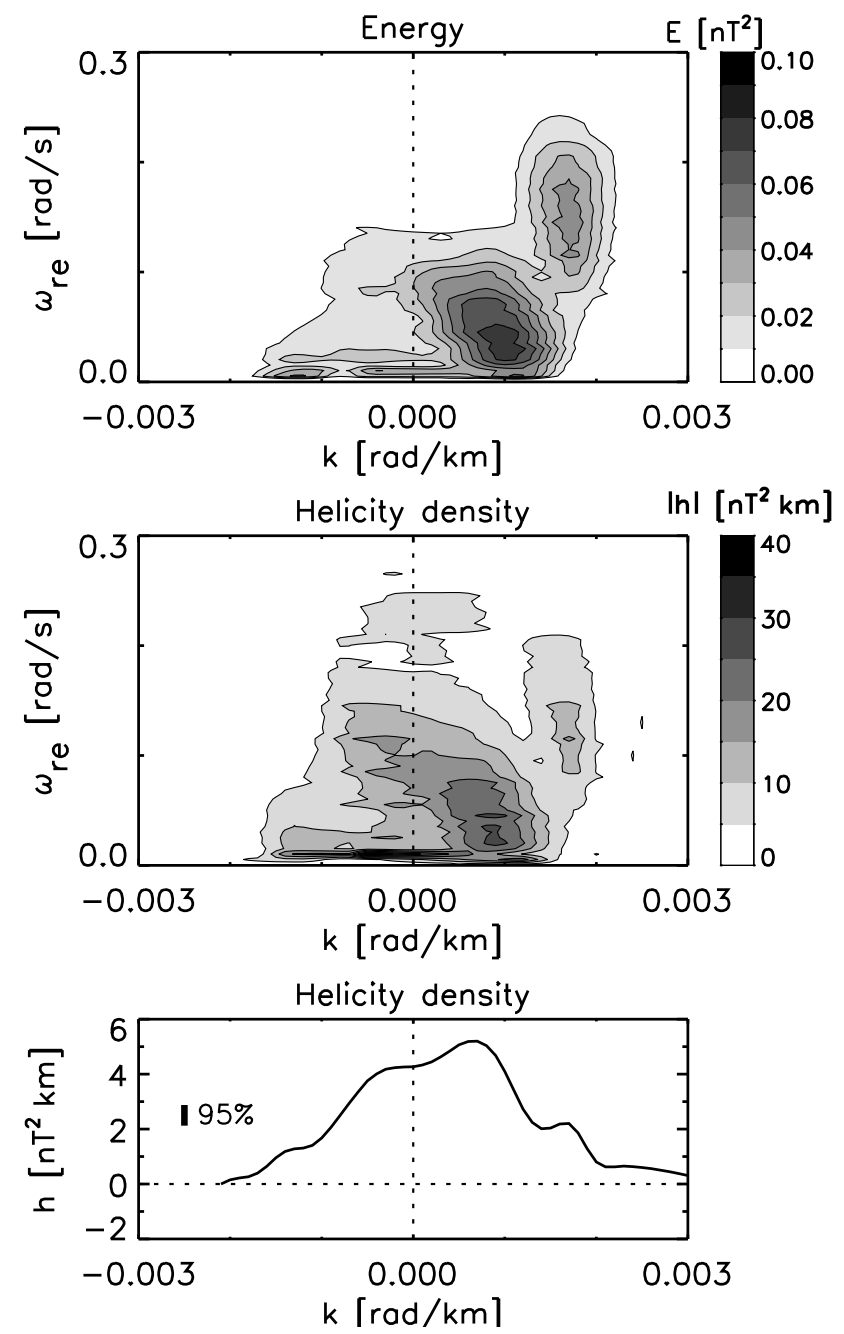

Fig. 8. Energy distribution (top) and magnetic helicity density (middle and bottom) for the foreshock wave observation of Cluster in the time interval on 18 February 2002, from 08:10 to 08:30 UT. Frequency and wave number (parallel to the mean magnetic field) are presented in the plasma rest frame. The vertical bar in the bottom panel is the $95 \%$ confidence interval.

meier, 2005), which justifies the choice of the parallel wave number for presentation of the energy and helicity distribution. The energy distribution exhibits two major peaks. One is located around the frequency $\omega_{r e}=0.04 \mathrm{rad} / \mathrm{s}$ and the wave number $k=0.0010 \mathrm{rad} / \mathrm{km}$. The second peak is found around the frequency $\omega_{r e}=0.15 \mathrm{rad} / \mathrm{s}$ and the wave number $k=0.0017 \mathrm{rad} / \mathrm{km}$. (Note that the analysis method is different from the one used in Narita et al. (2004): the energy is determined in the plasma rest frame in this manuscript and furthermore it is smoothed in the frequency and the wave number domain after the Doppler shift correction) Both peaks are located on the positive wave number side, parallel to the mean magnetic field direction and in the direction away 
from the shock to the interplanetary space. The phase speeds are therefore about $v_{p h}=40 \mathrm{~km} / \mathrm{s}$ and $v_{p h}=88 \mathrm{~km} / \mathrm{s}$ for the first and the second peak, respectively. The second peak is close to the Alfvén speed of the background plasma, about $77 \mathrm{~km} / \mathrm{s}$, while the first one is about half of the Alfvén speed.

The distribution of the magnetic helicity density in the $\omega-$ $k$ domain is displayed in the middle panel of Fig. 8. Since the helicity density can take both a positive and a negative value, magnitude of the helicity density is plotted here, too. The helicity density distribution again exhibits two peaks that are identified in the energy distribution, though the frequencies and the wave numbers at the peaks are slightly different: the first peak around the frequency $\omega_{r e}=0.03 \mathrm{rad} / \mathrm{s}$ and the wave number $k=0.0009 \mathrm{rad} / \mathrm{km}$, and the second around $\omega_{r e}=0.11 \mathrm{rad} / \mathrm{s}$ and the wave number $k=0.0017 \mathrm{rad} / \mathrm{km}$. The helicity density has an extended distribution from the first peak to the negative wave number regime, while the helicity density near the second peak is not large.

The helicity density is then averaged over the rest-frame frequencies to obtain the one-dimensional helicity density. The result is displayed in Fig. 8 bottom panel. The confidence interval with $95 \%$ confidence is also shown in the panel, which is determined by the degrees of freedom used in the wave telescope analysis. 100 degrees of freedom are used in the analysis.

An asymmetry in the helicity density is clearly seen between the parallel and the anti-parallel direction to the mean field. The helicity density is positive in the investigated wave number range, both in the parallel and anti-parallel directions. Larger values of the helicity density are found in the parallel direction than in the anti-parallel direction. The onedimensional helicity density exhibits the maximum at the wave number about $k=0.0008 \mathrm{rad} / \mathrm{km}$ which comes from the major peak identified in the two-dimensional energy and helicity distributions (top and middle panels). There are also other peaks in the one-dimensional helicity density. The peak at $k=0.0017 \mathrm{rad} / \mathrm{km}$ again comes from that already identified in the two-dimensional distributions. A minor peak at $k=-0.0014 \mathrm{rad} / \mathrm{km}$ originates from the helicity density population at the rest-frame frequencies nearly zero.

To summarize the analysis, the dominant waves (identified as the peaks in the energy distribution) are propagating parallel to the magnetic field in the plasma rest frame, which is in the direction from the Earth to the sun. The helicity density distribution confirms the dominance of the parallel propagation but on the other hand exhibits some anti-parallel propagation components (from the sun to the Earth). The helicity density is positive in the both directions. The dominant wave components are parallel-propagating and have the left-hand rotated sense of helicity, corresponding to positive polarization (right-hand temporal rotation about the magnetic field). There are minor, anti-parallel propagating wave components that have also the left-hand rotated sense of helicity. The anti-parallel propagating waves are therefore associated with negative polarization (left-hand temporal rotation about the magnetic field).

The characteristics of the dominant wave components are well explained by the right-hand resonant beam instability (Gary, 1993), and confirm the results of earlier Cluster data analyses (Narita et al., 2003; Narita and Glassmeier, 2005; Narita et al., 2007). The characteristics of the minor, antiparallel propagating waves can be explained by the nonresonant beam instability (Gary, 1993) or wave-wave interactions (Narita et al., 2007).

The helicity distribution in the $\omega-k$ domain reflects a lot the energy distribution. This is, however, not surprising because both the energy and the helicity density are the second order moment of probability distribution of fluctuation, and it is proportional to the square of fluctuation amplitude. The difference between the energy and the helicity density is that the former is a measure of variance (squared amplitude), while the latter is a measure of cross correlation between different components.

\section{Conclusions}

The magnetic helicity density can be determined in the wave number domain on the basis of multi-point measurements and it serves as a diagnostic tool of the spiral geometry of magnetic field lines. The numerical test successfully identified the wavelength and the rotation sense of the generated waves, though the estimated helicity densities are somewhat smaller than that of the analytical model. It is also found that the distribution of the magnetic helicity density resembles that of the energy qualitatively a lot. Using Cluster observations, the energy and the helicity profiles are uniquely determined in the plasma rest frame, which allows detailed comparisons with various theories of waves and instabilities.

If the magnetic field is composed of a weakly inhomogenous background field and random-like fluctuations, then the helicity density of the field fluctuations can be estimated independent of gauge over spatial and temporal scales that are much smaller than those of the background field. Also, it can be used as a wave diagnostic tool to quantify how the spiral geometry of magnetic field is configured on various spatial scales, whether the sense of field rotation is right- or lefthanded with respect to the propagation direction, and how large its magnitude is, though the estimate of the helicity density moderately depend on how the statistical analysis is done.

Many other applications are possible using the presented estimator of the magnetic helicity density. One of further applications of the helicity density measurements is the study of helicity transport in a turbulent medium. It is proposed that the magnetic helicity density is transported from one spatial scale to another, leading to a cascade of the helicity density. Magnetic field fluctuations in the solar wind are believed to be in a fully developed turbulent state, and it would 
be interesting to investigate how the magnetic helicity density distribution looks like in such a region.

Acknowledgements. This work was financially supported by Bundesministerium für Wirtschaft und Technologie and Deutsches Zentrum für Luft- und Raumfahrt, Germany, under contract 50 OC 0901.

Topical Editor R. Nakamura thanks S. C. Buchert and another anonymous referee for their help in evaluating this paper.

\section{References}

Angelopoulos, V.: The THEMIS Mission, Space Sci. Rev., 141, 534, 2008.

Auster, H.-U., Glassmeier, K.-H., Magnes, W., Aydogar, O., Baumjohann, W., Constantinescu, D., Fischer, D., Fornaçon, K.H., Georgescu, E., Harvey, P., Hillenmaier, O., Kroth, R., Ludlam, M., Narita, Y., Nakamura, R., Okrafka, K., Plaschke, F., Richter, I., Schwarzl, H., Stoll, B., Valavanoglou, A., and Wiedemann, M.: The THEMIS Fluxgate Magnetometer, Space Sci. Rev., 141, 235-264, 2008.

Balogh, A., Carr, C. M., Acua, M. H., Dunlop, M. W., Beek, T. J., Brown, P., Fornacon, K.-H., Georgescu, E., Glassmeier, K.H., Harris, J., Musmann, G., Oddy, T., and Schwingenschuh, K.: The Cluster Magnetic Field Investigation: overview of in-flight performance and initial results, Ann. Geophys., 19, 1207-1217, 2001 ,

http://www.ann-geophys.net/19/1207/2001/.

Berger, M. A. and Field, G. B.: The topological properties of magnetic helicity, J. Fluid Mech., 147, 133-148, 1984.

Biskamp, D.: Magnetohydrodynamics Turbulence, Cambridge University Press, Cambridge, 2003.

Escoubet, C. P., Fehringer, M., and Goldstein, M.: The Cluster mission, Ann. Geophys., 19, 1197-1200, 2001, http://www.ann-geophys.net/19/1197/2001/.

Frisch., U., Pouquet, A., Léorat, J., and Mazure, A.: Possibility of an inverse cascade of magnetic helicity in magnetohydrodynamic turbulence, J. Fluid Mech., 68, 769-778, 1975.

Gary, S. P.: Theory of Space Plasma Microinstabilities, Cambridge Atmospheric and Space Science Series, Cambridge University Press, 1993.

Glassmeier, K.-H., Coates, A. J., Acuña, M. H., Goldstein, M. L., Johnstone, A. D., Neubauer, F. M., and Rème, H.: Spectral characteristics of low-frequency plasma turbulence upstream of Comet P/Halley J. Geophys. Res., 94, 37-48, 1989.

Glassmeier, K.-H., Motschmann, U., Dunlop, M., Balogh, A., Acuña, M. H., Carr, C., Musmann, G., Fornaçon, K.-H., Schweda, K., Vogt, J., Georgescu, E., and Buchert, S.: Cluster as a wave telescope - first results from the fluxgate magnetometer, Ann. Geophys., 19, 1439-1447, 2001 (Correction, 21, 1071, 2003).

Matthaeus, W. H. and Goldstein, M. L.: Measurement of the rugged invariants of magnetohydrodynamic turbulence in the solar wind J. Geophys. Res., 87, 6011-6028, 1982.

Matthaeus, W. H., Goldstein, M. L., and Smith, C.: Evaluation of magnetic helicity in homogeneous turbulence, Phys. Rev. Lett., 48, 1256-1259, 1982.

Motschmann, U., Woodward, T. I., Glassmeier, K.-H., Southwood, D. J., and Pinçon, J. L.: Wavelength and direction filtering by magnetic measurements at satellite arrays: generalized minimum variance analysis, J. Geophys. Res., 101, 4961-4965, 1996.

Narita, Y., Glassmeier, K.-H., Schäfer, S., Motschmann, U., Sauer, K., Dandouras, I., Fornaçon, K.-H., Georgescu, E., and Rème, H.: Dispersion analysis of ULF waves in the foreshock using cluster data and the wave telescope technique, Geophys. Res. Lett., 30, SSC 43-1, doi:10.1029/2003GL017432, 2003.

Narita, Y., Glassmeier, K.-H., Schäfer, S., Motschmann, U., Fränz, M., Dandouras, I., Fornaçon, K.-H., Georgescu, E., Korth, A., Rème, H., and Richter, I.: Alfvén waves in the foreshock propagating upstream in the plasma rest frame: statistics from Cluster observations, Ann. Geophys., 22, 2315-2323, 2004, http://www.ann-geophys.net/22/2315/2004/.

Narita, Y. and Glassmeier, K.-H.: Dispersion analysis of lowfrequency waves through the terrestrial bow shock, J. Geophys. Res., 110, A12215, doi:10.1029/2005JA011256, 2005.

Narita, Y., Glassmeier, K.-H., Fränz, M., Nariyuki, Y., and Hada, T.: Observations of linear and nonlinear processes in the foreshock wave evolution, Nonlin. Processes Geophys., 14, 361-371, 2007, http://www.nonlin-processes-geophys.net/14/361/2007/.

Narita, Y., Glassmeier, K.-H., Dcrau, P. M. E., Hada, T., Motschmann, U., and Nariyuki, Y.: Evaluation of bispectrum in the wave number domain based on multi-point measurements, Ann. Geophys., 26, 3389-3393, 2008, http://www.ann-geophys.net/26/3389/2008/.

Pinçon, J. L. and Lefeuvre, F.: Local characterization of homogeneous turbulence in a space plasma from simultaneous measurements of field components at several points in space, J. Geophys. Res., 96, 1789-1802, 1991.

Rème, H., Aoustin, C., Bosqued, J. M., Dandouras, I., et al.: First multispacecraft ion measurements in and near the Earth's magnetosphere with the identical Cluster ion spectrometry (CIS) experiment, Ann. Geophys., 19, 1303-1354, 2001, http://www.ann-geophys.net/19/1303/2001/.

Smith, C. W., Goldstein, M. L., and Matthaeus, W. H.: Turbulence analysis of the Jovian upstream 'wave' phenomenon, J. Geophys. Res., 88, 5581, 1983; Correction, J. Geophys. Res., 89, 9159, 1984.

Subramanian, K. and Brandenburg, A.: Magnetic helicity density and its flux in weakly inhomogeneous turbulence, Astrophys. J., 648, L71-L74, 2006.

Stix, T. H.: The Theory of Plasma Waves, McGraw-Hill, New York, 1962.

Vogt, J., Narita, Y., and Constantinescu, O. D.: The wave surveyor technique for fast plasma wave detection in multi-spacecraft data, Ann. Geophys., 26, 1699-1710, 2008,

http://www.ann-geophys.net/26/1699/2008/.

Wiegelmann, T. and Büchner, J.: Evolution of magnetic helicity in the course of kinetic magnetic reconnection, Nonlin. Processes Geophys., 8, 127-140, 2001, http://www.nonlin-processes-geophys.net/8/127/2001/.

Wiegelmann, T. and Büchner, J.: Evolution of magnetic helicity in the course of kinetic magnetic reconnection: Part II $B \neq 0$ reconnection, Nonlin. Processes Geophys., 9, 139-147, 2002, http://www.nonlin-processes-geophys.net/9/139/2002/.

Woltjer, L.: A theorem on force-free magnetic fields, Proc. Nat. Acad. Sci. USA, 44, 489-492, 1958. 